\title{
Prevalence of Metabolic Syndrome Diagnosed by Three Different Criteria in School-Aged Children from Rural and Urban Areas of Northwest Mexico
}

\section{Cecilia Ramírez-Murillo, Elizabeth Guillot-Sánchez, Elizabeth Artalejo-Ochoa Q B, Alma E. Robles-Sardin, José A. Ponce-Martínez, María I Grijalva-Haro, Graciela Caire-Juvera, María I. Ortega-Vélez and Martha N. Ballesteros-Vásquez*}

Centro de Investigación en Alimentación y Desarrollo, A.C. Carretera a la Victoria km 0.6, Hermosillo, Sonora México

\begin{abstract}
The International Diabetes Federation (IDF) does not justify the evaluation of metabolic syndrome (MetS) in children aged less than 10 years, unless they have a family history of risk factors. The prevalence of overweight and obesity in the Northwest of Mexico has increased in recent decades, making it possible to consider that MetS is already present in this group of population.
\end{abstract}

Objective: The primary objective of this study was to determine the prevalence of metabolic syndrome in children aged 6 to 9 years living in rural (RA) or urban (UA) areas of Northwest of Mexico. A secondary objective was to find adequate criteria to diagnose the prevalence of MS in children.

Methods: Participated 268 school-aged children in a random-selected cross sectional study. Anthropometric and blood pressure measurement were performed, and biochemical indicators were analyzed. MetS was defined as the presence of three or more risk factors and diagnosed using three different criteria. One of them according to what was proposed by the International Diabetes Federation (IDF) for children and two additional criteria proposed by this study considering suitable cutoffs for age for lipids and blood pressure.

Results: The general prevalence of MetS according to the three different criteria used was as follows.1) IDF criteria, 4.1\%; 2) using cutoffs suggested for age for lipids and blood pressure and taking into account waist circumference as a criterion for MetS, 6.3\%; and 3) cutoffs suggested for age, lipids and blood pressure without considering waist circumference as a criterion for MetS, $10.4 \%$. Children living in the RA with a history of obesity and cardiovascular disease had higher waist circumference, triglycerides, and very low-density lipoprotein-C, and children from the UA had higher systolic and diastolic blood pressure, and higher levels of glucose and insulin.

Conclusion: MetS is present in children aged 6 to 9 years in the northwest region of Mexico, with higher proportions of the syndrome observed in overweight and obese children. The second criteria used in this study could be the most suitable for diagnosis of MetS, and the third criteria, for children at higher risk for cardiovascular disease and type 2 diabetes mellitus associated to heredity factors.

Keywords: Metabolic syndrome; Obesity; School-Aged Mexican children; Cardiovascular disease; Type 2 diabetes mellitus

\section{Introduction}

Overweight and obesity are highly associated with metabolic syndrome (MetS). This is defined as a clustering of metabolic abnormalities characterized by risk factors that are associated with cardiovascular disease and type 2 diabetes mellitus (T2DM). These factors are abdominal obesity, lipid profile alterations, glucose intolerance, and hypertension [1]. The presence of MetS increases the risk of developing cardiovascular disease and T2DM [2]. For adolescents, the evaluation criteria are not well defined and there is no accepted definition to diagnose MetS [3]. The International Diabetes Federation (IDF) [4]. does not justify the evaluation of MetS in children less than 10 years old, suggesting that the presence of MetS is out that its presence is unlikely in this population. Nevertheless, IDF states that more studies are needed to corroborate this statement.

One of the difficulties in evaluating MetS in children is the constant change in the level of insulin during childhood and the presence of physiological insulin resistance during puberty [5]. However, evidence indicates that people who develop diabetes in adulthood had higher body mass index (BMI) and subscapular skin fold as well as higher levels of glucose, triglycerides, and insulin, higher blood pressure, and lower levels of HDL-cholesterol (HDL-C) during childhood than those who do not develop diabetes [6]. Additionally, the American Pediatric Association suggests that cardiovascular risk factors be evaluated in children between 2 and 10 years of age when they have family history of cardiovascular diseases [7].
Currently, research on MetS focuses on identifying its components in different populations and at different ages as well as developing a useful definition for clinical and epidemiological practices. In addition to the traditional components of MetS, researchers are considering other indicators that could be helpful in identifying people at higher risk, such as acanthosis nigricans (AN) and inflammatory markers $[8,9]$.

In Mexico, T2DM and cardiovascular diseases are the main causes of mortality in the adult population [10]. Risk factors leading to these pathologies could be present during childhood mainly due to heredity or life style factors such as inadequate diets higher in carbohydrates or fat, together with low levels of physical activity $[11,12]$.

In this context and according to data from the National Health and Nutrition Survey in Mexico in 2012, the state of Sonora, located in the

*Corresponding author: Martha N. Ballesteros Vásquez, Centro de Investigación en Alimentación y Desarrollo, A.C. Carretera a la Victoria km 0.6, Hermosillo, Sonora México, Tel: +52 (662) 2892400 (ext. 297); E-mail: nydia@ciad.mx

Received July 30, 2015; Accepted August 19, 2015; Published August 24, 2015

Citation: Ramírez-Murillo C, Guillot-Sánchez E, Elizabeth Artalejo-Ochoa QB Robles-Sardin AE, Ponce-Martínez JA, et al. (2015) Prevalence of Metabolic Syndrome Diagnosed by Three Different Criteria in School-Aged Children from Rural and Urban Areas of Northwest Mexico. J Metabolic Synd 4: 181 doi:10.4172/2167-0943.1000181

Copyright: () 2015 Ramírez-Murillo C, et al. This is an open-access article distributed under the terms of the Creative Commons Attribution License, which permits unrestricted use, distribution, and reproduction in any medium, provided the original author and source are credited. 
Citation: Ramírez-Murillo C, Guillot-Sánchez E, Elizabeth Artalejo-Ochoa QB, Robles-Sardin AE, Ponce-Martínez JA, et al. (2015) Prevalence of Metabolic Syndrome Diagnosed by Three Different Criteria in School-Aged Children from Rural and Urban Areas of Northwest Mexico. $J$ Metabolic Synd 4: 181. doi:10.4172/2167-0943.1000181

northwest region of Mexico, is one of the states in the country with a higher prevalence of childhood overweight and obesity (36.9\%). Both rural and urban areas are affected, although in different ways; there are more overweight $(24.8 \%)$ than obese $(6.4 \%)$ children in rural areas, whereas the urban areas have more obese $(19.4 \%)$ than overweight (18.3\%) children [13]. Among the complications associated with excess body fat are insulin resistance [14], non-alcoholic fatty liver disease, hypertension, dyslipidemias, sleep disorders, orthopedic problems, and psychological and social issues [15].

The evaluation of MetS in children and adolescents is important for timely prevention and control of non-communicable disease development such as heart disease and T2DM. Therefore, the primary objective of this study was to determine the prevalence of metabolic syndrome in children aged 6 to 9 years living in rural and urban areas of Northwest of Mexico. A secondary objective was to compare MetS prevalence differences based on three criteria in a sample of children from Sonora, Mexico and determine the most appropriate criterion to diagnose it in a pediatric population.

\section{Materials and Methods}

\section{Study design and sample selection}

For this study, a cross-sectional sample of school-aged children aged 6 to 9 years was assessed. Eighteen public elementary schools located in two different areas (rural and urban) in the state of Sonora in northwest Mexico participated in the study. In the rural area, 7 schools from different towns were involved. In the urban area, 11 schools located in the city of Hermosillo (the capital) participated in the study. The rural and urban areas were defined according to the criteria established by Villalvazo [16], who defined a rural area as having less than 15,000 inhabitants. A total of 295 children participated in the study.

\section{Study protocol}

The experimental protocol was approved by the Ethical Review Board of Centro de Investigación en Alimentación y Desarrollo A.C. We used simple random sampling for the selection of the schools. Forty invitations were left in each school, inviting parents who had children between 6 and 9 years old to participate in the study. Parents and children who attended the invitation, received a detailed explanation of the protocol and provided informed consent. The parents answered a clinical questionnaire about family history of diabetes or cardiovascular risks, current medical conditions, and medication use.

A standard questionnaire was used to collect information about socio-demographic characteristics, and patient's medical and family history during face-to-face interviews conducted by trained staff. Family history of obesity, diabetes, and cardiovascular disease were defined as the existence of these conditions in at least one first-degree relative.

Anthropometric measurements were performed according to standard procedures. Body weight was measured using an electronic scale with a capacity of 0 to $150 \pm 0.05 \mathrm{~kg}$ (AND FV-150 KA1; A\&D Co. Japan), and height was measured using a portable stadiometer (Holtain Limited Dyfed, Britain, UK). BMI was calculated using weight and height $\left(\mathrm{kg} / \mathrm{m}^{2}\right)$ and $\mathrm{BMI}$ for age $\mathrm{z}$ score, height for age $\mathrm{z}$ score, and weight for age $\mathrm{z}$ score were calculated using the Anthro Plus software [17]. Obesity was defined following World Health Organization (WHO) criteria for children of the same age. Waist circumference (WC), was measured with non-elastic tape (Lafayette Instruments, USA) at the midpoint between the lower rib margin and the iliac crest, perpendicular to the long axis of the body, with the subject standing balanced on both feet. Body composition was measured using bioelectrical impedance analysis (Impedimed IMP5 ${ }^{\mathrm{TM}}$ ). Since we could not assess a hydration status of the children before measurement, we applied a brief clinical questionnaire to the parents in order to know if their children presented illness or diarrhea episodes 5 days before the evaluation. If the answer was positive, the child was not included in the study. Total body fat and lean mass were obtained using the RamirezLópez formula developed for the population of school children living in Sonora [18]. Body fat percentage was classified according to Freedman's values for age and gender [19].

Blood pressure was measured according to the technique proposed by the National High Blood Pressure Education Program (NHBPEP) for children and adolescents [20] after 5 minutes of rest in a sitting position with the child's feet on the floor and their arms supported at heart level. Two measurements were performed at 10-minute intervals using mercurial sphygmomanometers (Desk Model Mercurial Sphygmomanometer, Model 100, China Meheco Medical instrument, RPC) with an appropriate cuff for the arm diameter. Hypertension was defined as the average systolic (SBP) or diastolic (DBP) blood pressure level greater than or equal to the $95^{\text {th }}$ percentile for the child's sex, age, and height. AN was defined as a skin darkening and thickening at the neck [21]. The determination of AN child was assessed by a trained individual.

\section{Biochemical analyses}

Plasma obtained after overnight fasting was used to determine plasma glucose, total cholesterol (TC), HDL-C, triglycerides, and insulin. Two fasting (12-h) blood samples were collected from each subject on 2 different days. Blood was collected in tubes containing $0.15 \mathrm{~g} / 100 \mathrm{~g}$ EDTA to determine plasma lipids. Plasma was separated by centrifugation at $1500 \mathrm{~g}$ for $20 \mathrm{~min}$ at $4^{\circ} \mathrm{C}$ then placed into vials containing phenyl methyl sulfonyl fluoride $(0.015 \mathrm{~g} / 100 \mathrm{~g})$, sodium azide $(0.01 \mathrm{~g} / 100 \mathrm{~g})$, and aprotinin $(0.01 \mathrm{~g} / 100 \mathrm{~g})$.

TC was determined using an enzymatic method (CHOD-PAO) with Roche-Diagnostic standards and kits [22]. HDL-C was measured in supernatant after precipitation of apo B-containing lipoproteins [23], and low density lipoprotein (LDL)-C was determined using the Friedwald equation [24]. Triglycerides were measured using an enzymatic method (GPO-PAP) with Roche-Diagnostics standards and kits [25]. Means of two blood draws were used.

Plasma glucose was determined using an enzymatic method (GOD-PAO) with Roche Diagnostic kits [26]. Fasting plasma insulin concentration was determined by enzyme-linked immunosorbent assay (ELISA) using a sandwich type immunoassay (ALPCO Diagnostics, $\mathrm{NH}, \mathrm{USA}$ ). The homeostasis model assessment (HOMA) [27] was used to calculate insulin resistance (IR) according to the following equation: IR (HOMA IR) - fasting insulin $(\mu \mathrm{U} / \mathrm{mL})$ fasting glucose $(\mathrm{mmol} / \mathrm{L})$ $\div 22.5$. The HOMA model has been shown to be a reliable method for measuring insulin resistance in various populations when other more invasive methods are not feasible [28]. The HOMA-IR value of 3.4 was chosen as the reference value to define IR [28] and the insulin blood concentration was classified as normal, borderline, or high [29].

\section{Definition of metabolic syndrome}

Because there is no single accepted criteria for the diagnosis of MetS in children aged 6 to 9 years, the presence of MetS in this population was determined using three different criteria as follows: 1) according to the definition proposed by IDF [4] for children aged 10 years, considering the presence of central obesity as a mandatory condition for diagnosis coupled with the presence of two or more additional risk 
Citation: Ramírez-Murillo C, Guillot-Sánchez E, Elizabeth Artalejo-Ochoa QB, Robles-Sardin AE, Ponce-Martínez JA, et al. (2015) Prevalence of Metabolic Syndrome Diagnosed by Three Different Criteria in School-Aged Children from Rural and Urban Areas of Northwest Mexico. $J$ Metabolic Synd 4: 181. doi:10.4172/2167-0943.1000181

Page 3 of 9

factors; 2) considering the presence of central obesity according to the provisions of the IDF [4], but using predefined cutoffs for lipids specific for children [30] and blood pressure [20]; 3) establishing the presence of three or more risk factors regardless of central obesity as a mandatory condition and using predefined cutoffs determined by the IDF [4] for waist circumference and glucose, the National Cholesterol Education Program (NCEP) criteria [30] for lipids, and the NHBPEP criteria [20] for blood pressure (Table 1).

Central obesity was defined as a waist circumference value at or above the $90^{\text {th }}$ percentile for sex, age, and ethnicity from the IDF definition [4]. For the lipid profile, we considered the cut off values from the National Cholesterol Education Program [30]. Triglycerides, LDL-C, and TC were considered elevated when values were at or above the $95^{\text {th }}$ percentile for age and sex, and HDL-C was considered low when the values were at or under the $5^{\text {th }}$ percentile [30]. Elevated SBP or DBP were defined as values at or above the $95^{\text {th }}$ percentile for age, sex, and height [20]. The reference value for impaired fasting glucose was taken from the IDF and considered as $100 \mathrm{mg} / \mathrm{dL}$ or higher.

\section{Statistical analysis}

Normality of the data was verified and descriptive statistics were performed to show the study population characteristics by rural/ urban area. Data are presented as means \pm standard deviations and as medians and interquartile ranges accordingly. Normality and equality of variances were evaluated and the tests were used accordingly. Twosample Student's t-test was used to compare both areas of study. For non-normal variables, we used the Mann Whitney U Test. For nonequal variances, the Aspin Welch p value was selected. The Chi-squared test for Independence was used to compare frequencies. Differences with $\mathrm{p}<0.05$ were considered significant. The kappa coefficient was used to test the level of agreement for the three definitions. All data were analyzed using NCSS 2007 (Number Cruncher Statistical System for Windows, Kaysville, Utah, USA) Software.

\section{Results}

Children's anthropometric characteristics by area (rural and urban) are presented in Table 2 . The mean age of the children was $7.4 \pm 1.03$

\begin{tabular}{|c|c|c|c|}
\hline Risk factor & IDF, 2007 & $\begin{array}{l}\text { IDF (2007), NCEP } \\
\text { (1991), NHBPEP } \\
(2005) \\
\text { (proposed in this } \\
\text { study) }\end{array}$ & $\begin{array}{l}\text { Three or more } \\
\text { risk factors NCEP } \\
\text { (1991), NHBPEP } \\
\text { (2005) without WC } \\
\text { as a condition. } \\
\text { (proposed in this } \\
\text { study) }\end{array}$ \\
\hline Obesity & $\begin{array}{l}\text { Obligatory } \\
\text { presence of waist } \\
\text { circumference } \geq 90^{\text {th }} \\
\text { percentile for age } \\
\text { and sex } \\
+2 \text { risk factors }\end{array}$ & $\begin{array}{l}\text { Obligatory } \\
\text { presence of waist } \\
\text { circumference } \\
\geq 90^{\text {th }} \text { percentile for } \\
\text { age and sex }[4] \\
+2 \text { risk factors }\end{array}$ & $\begin{array}{l}\text { Waist circumference } \\
\text { in } \geq 90^{\text {th }} \text { percentile } \\
\text { for age and sex [4] }\end{array}$ \\
\hline $\begin{array}{l}\text { Triglycerides } \\
(\mathrm{mg} / \mathrm{dL})\end{array}$ & $\geq 150$ & $\begin{array}{l}\geq 95^{\text {th }} \text { percentile for } \\
\text { age and sex [28] }\end{array}$ & $\begin{array}{l}\geq 95^{\text {th }} \text { percentile for } \\
\text { age and sex [28] }\end{array}$ \\
\hline $\begin{array}{l}\text { Glucose } \\
\text { (mg/dL) }\end{array}$ & $\geq 100$ & $\geq 100 \mathrm{mg} / \mathrm{dL}$ [4] & $\geq 100 \mathrm{mg} / \mathrm{dL}[4]$ \\
\hline $\begin{array}{l}\mathrm{HDL}-\mathrm{C} \\
(\mathrm{mg} / \mathrm{dL})\end{array}$ & $<40$ & $\begin{array}{l}\leq 5^{\text {th }} \text { percentile for } \\
\text { age and sex [28] }\end{array}$ & $\begin{array}{l}\leq 5^{\text {th }} \text { percentile for } \\
\text { age and sex [28] }\end{array}$ \\
\hline $\begin{array}{l}\text { SBP and/ } \\
\text { or DBP }(\mathrm{mm} \\
\mathrm{Hg})\end{array}$ & $\begin{array}{l}\text { Systolic } \geq 130 \\
\text { Diastolic } \geq 85\end{array}$ & $\begin{array}{l}\geq 95^{\text {th }} \text { percentile for } \\
\text { age, sex and height } \\
\text { [18] }\end{array}$ & $\begin{array}{l}\geq 95^{\text {th }} \text { percentile for } \\
\text { age, sex and height } \\
\text { [18] }\end{array}$ \\
\hline
\end{tabular}

Abbreviations: HDL-C: High Density Lipoprotein Cholesterol, IDF: International Diabetes Federation, NCEP: National Cholesterol Education Program, NHBPEP: National High Blood Pressure Education Program, SBP: Systolic Blood Pressure, DBP: Diastolic Blood Pressure.

Table 1: Criteria used to diagnose metabolic syndrome.

\begin{tabular}{|c|c|c|c|c|}
\hline Indicator & $\begin{array}{l}\text { Total } \\
(n=268)\end{array}$ & $\begin{array}{l}\text { Rural area } \\
(n=119)\end{array}$ & $\begin{array}{l}\text { Urban area } \\
(n=149)\end{array}$ & $\mathbf{p}^{1}$ \\
\hline Age (years) ${ }^{*}$ & $\begin{array}{l}7.5 \\
(1.4-3.9)\end{array}$ & $\begin{array}{l}7.6 \\
(1.3-3.7)\end{array}$ & $\begin{array}{l}7.3 \\
(1.45-3.9)\end{array}$ & 0.54 \\
\hline Weight $(\mathrm{Kg})^{*}$ & $\begin{array}{l}24.59 \\
(8.86-44.65)\end{array}$ & $\begin{array}{l}25.29 \\
(7.8-44.65)\end{array}$ & $\begin{array}{l}24.14 \\
(10.17-31.05)\end{array}$ & 0.25 \\
\hline Height $(\mathrm{cm})^{\dagger}$ & $\begin{array}{l}124.05 \pm 7.30 \\
(105.2-144.4)\end{array}$ & $\begin{array}{l}124.76 \pm 7.13 \\
(105.2-144.4)\end{array}$ & $\begin{array}{l}123.48 \pm 7.40 \\
(108.5-140.9)\end{array}$ & 0.15 \\
\hline z-BMI/A* & $\begin{array}{l}0.015 \\
(2.07-8.33)\end{array}$ & $\begin{array}{l}0.12 \\
(2.02-7.49)\end{array}$ & $\begin{array}{l}-0.06 \\
(2.27-7.87)\end{array}$ & 0.15 \\
\hline $\mathrm{z}-\mathrm{H} / \mathrm{A}^{\dagger}$ & $\begin{array}{l}0.006 \pm 0.99 \\
(-2.54-2.56)\end{array}$ & $\begin{array}{l}0.093 \pm 0.99 \\
(-2.54-2.54)\end{array}$ & $\begin{array}{l}-0.06 \pm 0.99 \\
(-2.33-2.56)\end{array}$ & 0.20 \\
\hline$z-W / A^{*}$ & $\begin{array}{l}0.21 \\
(2.06-8.11)\end{array}$ & $\begin{array}{l}0.28 \\
(1.81-7.72)\end{array}$ & $\begin{array}{l}0.14 \\
(2.19-7.04)\end{array}$ & 0.38 \\
\hline$W C(\mathrm{~cm})$ * & $\begin{array}{l}55.4 \\
(10.57-55.5)\end{array}$ & $\begin{array}{l}56.4 \\
(10.1-50.6)\end{array}$ & $\begin{array}{l}55.0 \\
(10.5-45.3)\end{array}$ & 0.21 \\
\hline$\%$ fat mass $(\mathrm{BIA})^{\dagger}$ & $\begin{array}{l}28.06 \pm 8.93 \\
(8.49-50.22)\end{array}$ & $\begin{array}{l}27.49 \pm 8.78 \\
(11.46-49.70)\end{array}$ & $\begin{array}{l}28.52 \pm 9.05 \\
(8.49-50.22)\end{array}$ & 0.35 \\
\hline Triglycerides $(\mathrm{mg} / \mathrm{dL})$ * & $\begin{array}{l}88.29 \\
(44.38-341.0)\end{array}$ & $\begin{array}{l}92.04 \\
(40.75-174.95)\end{array}$ & $\begin{array}{l}84.55 \\
(45.67-341.0)\end{array}$ & 0.01 \\
\hline $\mathrm{TC}(\mathrm{mg} / \mathrm{dL})^{\dagger}$ & $\begin{array}{l}170.82 \pm 33.40 \\
(86.07-304.92)\end{array}$ & $\begin{array}{l}172.23 \pm 33.79 \\
(86.78-304.92)\end{array}$ & $\begin{array}{l}169.69 \pm 33.15 \\
(86.07-277.15)\end{array}$ & 0.53 \\
\hline $\mathrm{HDL}-\mathrm{C}(\mathrm{mg} / \mathrm{dL})$ * & $\begin{array}{l}48.71 \\
(11.89-96.68)\end{array}$ & $\begin{array}{l}51.27 \\
(13.07-96.68)\end{array}$ & $\begin{array}{l}47.01 \\
(11.59-60.3)\end{array}$ & 0.01 \\
\hline LDL-C (mg/dL) * & $\begin{array}{l}101.12 \\
(43.54-194.01)\end{array}$ & $\begin{array}{l}103.06 \\
(39.32-189.08)\end{array}$ & $\begin{array}{l}99.8 \\
(48.40-169.95)\end{array}$ & 0.61 \\
\hline $\operatorname{VLDL}(\mathrm{mg} / \mathrm{dL}){ }^{*}$ & $\begin{array}{l}17.66 \\
(8.87-68.2)\end{array}$ & $\begin{array}{l}18.41 \\
(8.15-34.99)\end{array}$ & $\begin{array}{l}16.91 \\
(9.13-68.2)\end{array}$ & 0.01 \\
\hline $\operatorname{SBP}(\mathrm{mmHg}) *$ & $\begin{array}{l}90 \\
(20-70)\end{array}$ & $\begin{array}{l}90 \\
(20-70)\end{array}$ & $\begin{array}{l}90 \\
(15-60)\end{array}$ & 0.30 \\
\hline $\mathrm{DBP}(\mathrm{mmHg})$ * & $\begin{array}{l}60 \\
(17.5-67.5)\end{array}$ & $\begin{array}{l}60 \\
(17.5-52.5)\end{array}$ & $\begin{array}{l}60 \\
(18.75-65)\end{array}$ & 0.60 \\
\hline $\begin{array}{l}\text { Fasting glucose (mg/ } \\
\mathrm{dL})^{\dagger}\end{array}$ & $\begin{array}{l}93.29 \pm 13.99 \\
(44.82-130.21)\end{array}$ & $\begin{array}{l}89.86 \pm 14.40 \\
(44.82-126.64)\end{array}$ & $\begin{array}{l}96.04 \pm 13.05 \\
(61.65-130.21)\end{array}$ & $<0.01$ \\
\hline Insulin $(\mathrm{mU} / \mathrm{L}){ }^{*}$ & $\begin{array}{l}6.24 \\
(6.21-62.51)\end{array}$ & $\begin{array}{l}5.32 \\
(5.41-38.95)\end{array}$ & $\begin{array}{l}7.27 \\
(6.97-62.51)\end{array}$ & 0.03 \\
\hline HOMA* $^{*}$ & $\begin{array}{l}1.4 \\
(1.53-15.02)\end{array}$ & $\begin{array}{l}1.22 \\
(1.17-10.21)\end{array}$ & $\begin{array}{l}1.70 \\
(1.83-15.02)\end{array}$ & $<0.01$ \\
\hline AN, n (\%) $)^{\star \star}$ & $15(5.6)$ & $9(7.6)$ & $6(4)$ & 0.21 \\
\hline
\end{tabular}

${ }^{\dagger}$ Mean \pm standard deviation (minimum - maximum).

*Median (interquartile range [p25-p75]).

'Difference between groups by two sample Student's t-test and Mann Whitney U Test for non-normal data $(\mathrm{p}<0.05)$. ${ }^{*}$ Chi-squared test.

Abbreviations: z-BMI/A z: score of body mass index for age, z-H/A z: score height for age, $z-W / A$ z: score weight for age, SBP: systolic blood pressure, DBP: diastolic blood pressure, AN: acanthosis nigricans, WC: waist circumference, HDL-C: high density lipoprotein cholesterol, TC: total cholesterol, LDL-C: low density lipoprotein, VLDL: very low density lipoprotein, HOMA: Homeostasis Model Assessment.

Table 2: Anthropometric characteristics and metabolic profile of the total population and by rural or urban area.

years, ranging from 5 to 10 years. No statistically significant differences were observed between rural and urban areas in the anthropometric variables, blood pressure and the AN indicator $(p>0.05)$. According to the $\mathrm{z}$ BMI/A, the prevalence of overweight and obesity was $31.1 \%$ in rural areas and $32.9 \%$ in urban areas. Waist circumference was at or above the $90^{\text {th }}$ percentile in $10.1 \%$ of children in rural areas and $12 \%$ in urban areas. Likewise, children with moderate and high body fat percentage were observed in both study areas: $26 \%$ and $33 \%$ in rural and $27 \%$ and $36 \%$ in urban areas, respectively.

There were statistically significant differences between the urban and rural areas in some metabolic variables. Fasting blood glucose, insulin, and the HOMA index were higher in the urban area, whereas concentrations of triglycerides, HDL-C, and very low density lipoprotein (VLDL)-C were higher in the rural area (Table 2). 
Citation: Ramírez-Murillo C, Guillot-Sánchez E, Elizabeth Artalejo-Ochoa QB, Robles-Sardin AE, Ponce-Martínez JA, et al. (2015) Prevalence of Metabolic Syndrome Diagnosed by Three Different Criteria in School-Aged Children from Rural and Urban Areas of Northwest Mexico. J Metabolic Synd 4: 181. doi:10.4172/2167-0943.1000181

Page 4 of 9

According to the American Diabetes Association [31], fasting glucose values in children with normal, impaired, and probable diagnosis of diabetes are $86.1 \mathrm{mg} / \mathrm{dL}, 108.5 \mathrm{mg} / \mathrm{dL}$, and $128.4 \mathrm{mg} / \mathrm{dL}$ $(\mathrm{p}<0.01)$, respectively. Figure 1a shows the classification of the study population according to their blood glucose concentration.

Glucose data were divided according to normal, borderline, and high insulin blood levels. There were no differences in concentrations of glucose $(\mathrm{p}=0.99)$ relative to insulin levels, even when we found borderline and high values of insulin (Figure 1b).

According to the HOMA index, insulin resistance was present in 9 children from the rural area (7.5\%) and 22 from the urban area (14.8\%). There were no statistically significant differences between study groups $(\mathrm{p}=0.06)$. AN, which is associated with high levels of insulin, was found in 15 children including 9 from the rural and 6 from the urban areas (Table 2). This indicator was observed only in children with a high body fat mass.

Results of the anthropometric and metabolic characteristics in the population stratified by family history of obesity, diabetes, and cardiovascular disease are shown in Table 3. Children with a history of obesity had a significantly higher weight/age (W/A) and percentage of fat mass compared to children with no familiar history of obesity. Those with a history of diabetes had higher BMI/A and waist circumference, and children from families with a history of cardiovascular disease had higher systolic blood pressure, DBP, TC, and LDL-C compared with children without a family history of cardiovascular disease. Particularly in the rural area, a family history of obesity and cardiovascular diseases was present in children with a higher waist circumference ( 58.5 vs. 53.8 $\mathrm{cm}, \mathrm{p}<0.02$; 59 vs. $54.2 \mathrm{~cm}, \mathrm{p}<0.01)$. In contrast, urban children had higher SBP ( $94.46 \pm 11.97$ vs. $88.17 \pm 10.98 \mathrm{mmHg}, \mathrm{p}<0.001)$ and DBP $(63.36 \pm 12.92$ vs $57.60 \pm 10.08 \mathrm{mmHg} ; \mathrm{p}<0.001)$ when a family history of obesity and cardiovascular diseases was present.

Table 4 shows the proportion of children with MetS according to the different definitions used in the present study. For the first criteria suggested by the IDF [4], MetS was present in 11 children (4.1\%). In the rural and urban areas, $2.5 \%$ and $5.4 \%$ of the children had MetS,

a)

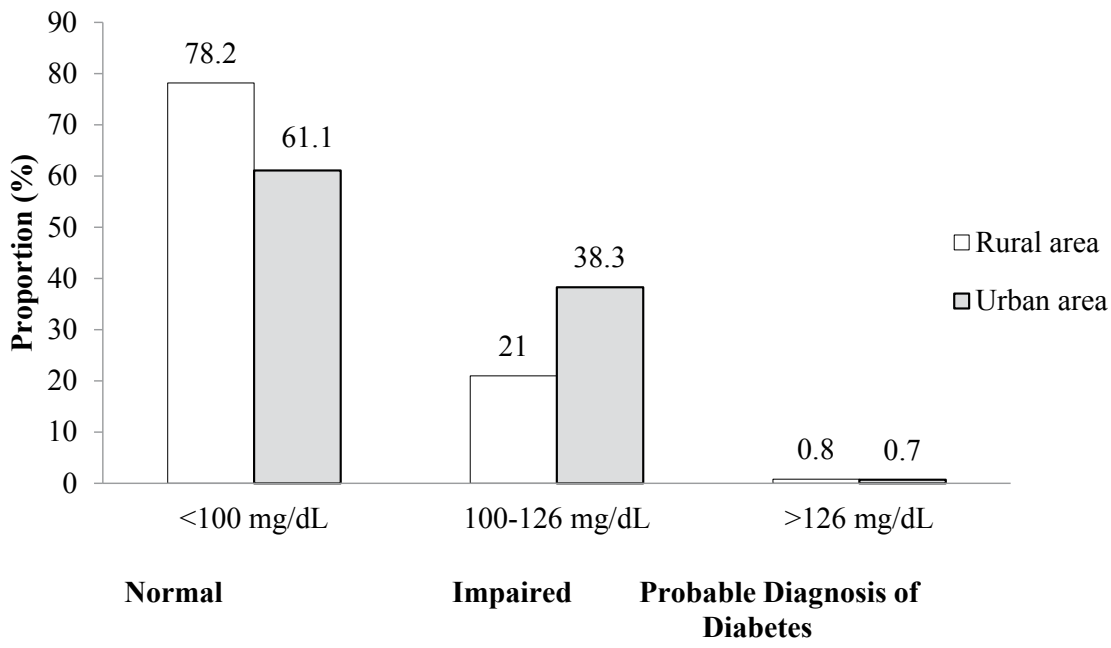

b)

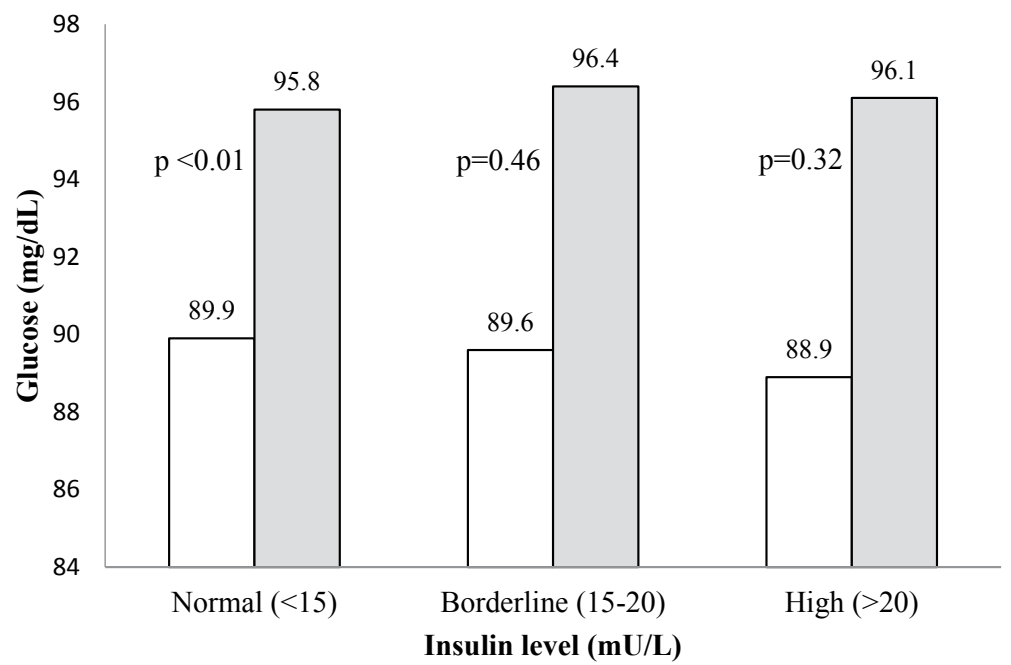

$\square$ Rural area

口Urban area

blood glucose concentration. Abbreviations: PDD Probable diagnosis of diabetes. 
Citation: Ramírez-Murillo C, Guillot-Sánchez E, Elizabeth Artalejo-Ochoa QB, Robles-Sardin AE, Ponce-Martínez JA, et al. (2015) Prevalence of Metabolic Syndrome Diagnosed by Three Different Criteria in School-Aged Children from Rural and Urban Areas of Northwest Mexico. $J$ Metabolic Synd 4: 181. doi:10.4172/2167-0943.1000181

Page 5 of 9

\begin{tabular}{|c|c|c|c|c|c|c|c|c|c|}
\hline Indicator & $\begin{array}{l}\text { Without obesity } \\
\qquad(n=44)\end{array}$ & $\begin{array}{l}\text { Obesity } \\
(n=90)\end{array}$ & $\mathbf{p}^{1}$ & $\begin{array}{c}\text { Without diabetes } \\
\quad(n=93)\end{array}$ & $\begin{array}{l}\text { Diabetes } \\
(n=18)\end{array}$ & $\mathbf{p}^{1}$ & $\begin{array}{l}\text { Without CVD } \\
\quad(n=63)\end{array}$ & $\begin{array}{c}\text { CVD } \\
(n=25)\end{array}$ & $\mathbf{p}^{1}$ \\
\hline Age (years) & $\begin{array}{c}7.5 \pm 0.7^{\dagger} \\
(6.1-9)\end{array}$ & $\begin{array}{c}7.4 \pm 0.9 \\
(6-9.7)\end{array}$ & 0.58 & $\begin{array}{c}7.3 \pm 0.9^{\dagger} \\
(6-9.7)\end{array}$ & $\begin{array}{l}7.5 \pm 0.7 \\
(6.1-8.4)\end{array}$ & 0.47 & $\begin{array}{c}7.33 \pm 0.73^{\dagger} \\
(6.1-8.8)\end{array}$ & $\begin{array}{c}7.46 \pm 0.94 \\
(6.1-9.7)\end{array}$ & 0.49 \\
\hline Weight $(\mathrm{Kg})$ & $\begin{array}{c}25.9 \pm 7.1^{\dagger} \\
(16.1-43)\end{array}$ & $\begin{array}{l}28.36 \pm 8.1 \\
(16.3-60.7)\end{array}$ & 0.10 & $\begin{array}{c}24.9^{*} \\
(10.6-44.6)\end{array}$ & $\begin{array}{c}28.1 \\
(10.6-32)\end{array}$ & 0.04 & $\begin{array}{c}25.4^{*} \\
(9.3-30.2)\end{array}$ & $\begin{array}{c}27.9 \\
(11.4-34.2)\end{array}$ & 0.34 \\
\hline Height (cm) & $\begin{array}{c}123.75 \pm 7.2^{\dagger} \\
(108.8-136.9)\end{array}$ & $\begin{array}{c}125.14 \pm 7.3 \\
(108.5-144.4)\end{array}$ & 0.29 & $\begin{array}{c}123.7 \pm 7.9^{\dagger} \\
(108.5-144.4)\end{array}$ & $\begin{array}{c}126.7 \pm 5.7 \\
(116.1-134.4)\end{array}$ & 0.16 & $\begin{array}{c}124.24 \pm 5.9^{\dagger} \\
(111.8-134.4)\end{array}$ & $\begin{array}{c}124.45 \pm 7.9 \\
(108.9-144.4)\end{array}$ & 0.89 \\
\hline z-BMI/A & $\begin{array}{c}0.34 \pm 1.7^{\dagger} \\
(-1.8-4.7)\end{array}$ & $\begin{array}{l}0.86 \pm 1.6 \\
(-2.8-5.5)\end{array}$ & 0.08 & $\begin{array}{c}0.48 \pm 1.5^{\dagger} \\
(-2.8-4.2)\end{array}$ & $\begin{array}{l}1.31 \pm 1.7 \\
(-0.9-5.5)\end{array}$ & 0.04 & $\begin{array}{c}0.60 \pm 1.44^{\dagger} \\
(-1.9-3.7)\end{array}$ & $\begin{array}{l}1.1 \pm 1.83 \\
(-1.4-5.5)\end{array}$ & 0.17 \\
\hline $\mathrm{Z}-\mathrm{H} / \mathrm{A}$ & $\begin{array}{c}0.03 \pm 1.0^{\dagger} \\
(-2.0-2.0)\end{array}$ & $\begin{array}{c}0.3 \pm 0.9 \\
(-2.2-2.56)\end{array}$ & 0.06 & $\begin{array}{c}0.13 \pm 0.9^{\dagger} \\
(-2.0-2.5)\end{array}$ & $\begin{array}{l}0.45 \pm 0.7 \\
(-0.96-1.8)\end{array}$ & 0.23 & $\begin{array}{c}0.2 \pm 0.85^{\dagger} \\
(-1.9-2.5)\end{array}$ & $\begin{array}{c}0.08 \pm 1.07 \\
(-2.0-2.0)\end{array}$ & 0.60 \\
\hline$z-W / A$ & $\begin{array}{l}0.3 \pm 1.6^{\dagger} \\
(-2.3-4.1)\end{array}$ & $\begin{array}{l}0.9 \pm 1.5 \\
(-2.3-5.3)\end{array}$ & 0.03 & $\begin{array}{c}0.46 \pm 1.5^{\dagger} \\
(-2.3-4.8)\end{array}$ & $\begin{array}{l}1.32 \pm 1.5 \\
(-0.7-5.3)\end{array}$ & 0.04 & $\begin{array}{c}0.59 \pm 1.3^{\dagger} \\
(-2.1-3.8)\end{array}$ & $\begin{array}{l}0.88 \pm 1.7 \\
(-2.3-5.3)\end{array}$ & 0.39 \\
\hline WC $(\mathrm{cm})$ & $\begin{array}{c}57.2 \pm 9.1^{\dagger} \\
(44.5-80)\end{array}$ & $\begin{array}{l}60.5 \pm 10.3 \\
(45.5-96.6)\end{array}$ & 0.08 & $\begin{array}{c}55.6^{*} \\
(11.2-50.6)\end{array}$ & $\begin{array}{c}59.7 \\
(15.9-36.1)\end{array}$ & 0.02 & $\begin{array}{c}56.6^{*} \\
(9.8-39.6)\end{array}$ & $\begin{array}{c}58.5 \\
(14.6-39.3)\end{array}$ & 0.35 \\
\hline $\begin{array}{l}\% \text { fat mass } \\
\text { (BIA) }\end{array}$ & $\begin{array}{c}26.9 \pm 9.1^{\dagger} \\
(13-48.3)\end{array}$ & $\begin{array}{c}30.5 \pm 9.5 \\
(11.5-50.2)\end{array}$ & 0.03 & $\begin{array}{l}28.1 \pm 9.0^{\dagger} \\
(11.5-50.2)\end{array}$ & $\begin{array}{c}31.2 \pm 10.1 \\
(13-49.3)\end{array}$ & 0.32 & $\begin{array}{c}28.47 \pm 9.2^{\dagger} \\
(11.5-49.5)\end{array}$ & $\begin{array}{c}30.8 \pm 2.0 \\
(15.8-49.3)\end{array}$ & 0.29 \\
\hline $\begin{array}{l}\text { Triglycerides } \\
(\mathrm{mg} / \mathrm{dL})\end{array}$ & $\begin{array}{l}98.6 \pm 57.3^{\dagger} \\
(47.8-378.5)\end{array}$ & $\begin{array}{c}99.9 \pm 34.4 \\
(37.5-193)\end{array}$ & 0.86 & $\begin{array}{c}89.2^{*} \\
(42.6-225.5)\end{array}$ & $\begin{array}{c}123.4 \\
(56.7-310.3)\end{array}$ & 0.19 & $\begin{array}{c}86.9^{*} \\
(41.2-341)\end{array}$ & $\begin{array}{c}102.3 \\
(58.4-129.5)\end{array}$ & 0.37 \\
\hline $\mathrm{TC}(\mathrm{mg} / \mathrm{dL})$ & $\begin{array}{c}175 \pm 33.3^{\dagger} \\
(86.8-277.1)\end{array}$ & $\begin{array}{l}173.8 \pm 37.2 \\
(86.1-304.9)\end{array}$ & 0.86 & $\begin{array}{c}179.43 \pm 32.6^{\dagger} \\
(86.8-304.9)\end{array}$ & $\begin{array}{l}159.6 \pm 21.7 \\
(129.3-76.8)\end{array}$ & 0.01 & $\begin{array}{c}168.79 \pm 35.6^{\dagger} \\
(86.1-251.7)\end{array}$ & $\begin{array}{l}185.36 \pm 33.9 \\
(142.4-277.1)\end{array}$ & 0.04 \\
\hline $\mathrm{HDL}-\mathrm{C}(\mathrm{mg} / \mathrm{dL})$ & $\begin{array}{c}47.14 \pm 10.7^{\dagger} \\
(19.2-70.1)\end{array}$ & $\begin{array}{c}51.3 \pm 12.1 \\
(8-104.7)\end{array}$ & 0.05 & $\begin{array}{c}50.6^{*} \\
(11.7-81)\end{array}$ & $\begin{array}{c}46.3 \\
(10.7-71.5)\end{array}$ & 0.36 & $\begin{array}{c}51.3^{*} \\
(12.5-96.7)\end{array}$ & $\begin{array}{c}50.2 \\
(14.6-48.4)\end{array}$ & 0.64 \\
\hline LDL-C (mg/dL) & $\begin{array}{c}108.1 \pm 31.3^{\dagger} \\
(37.4-200.2)\end{array}$ & $\begin{array}{l}102.5 \pm 34.0 \\
(30.2-224.2)\end{array}$ & 0.36 & $\begin{array}{l}108.9 \pm 30.5 \\
(37.4-186.9)\end{array}$ & $\begin{array}{c}87.6 \pm 20.8 \\
(47.9-126.3)\end{array}$ & 0.00 & $\begin{array}{c}98.18 \pm 33.0^{\dagger} \\
(30.2-179.3)\end{array}$ & $\begin{array}{c}114.22 \pm 30.7 \\
(78.8-200.2)\end{array}$ & 0.03 \\
\hline VLDL (mg/dL) & $\begin{array}{c}19.7 \pm 11.5^{\dagger} \\
(9.6-75.7)\end{array}$ & $\begin{array}{l}19.9 \pm 6.9 \\
(7.5-38.6)\end{array}$ & 0.86 & $\begin{array}{c}17.8^{*} \\
(8.5-45.1)\end{array}$ & $\begin{array}{c}24.7 \\
(11.3-62)\end{array}$ & 0.19 & $\begin{array}{c}17.4^{*} \\
(8.2-68.2)\end{array}$ & $\begin{array}{c}20.5 \\
(11.7-25.9)\end{array}$ & 0.37 \\
\hline $\mathrm{SBP}(\mathrm{mmHg})$ & $\begin{array}{c}90^{*} \\
(10-55)\end{array}$ & $\begin{array}{c}90 \\
(15-55)\end{array}$ & 0.82 & $\begin{array}{c}92.3 \pm 11.3^{\dagger} \\
(70-130)\end{array}$ & $\begin{array}{c}98.7 \pm 15.8 \\
(75-127.5)\end{array}$ & 0.03 & $\begin{array}{c}90^{*} \\
(15-50)\end{array}$ & $\begin{array}{c}90 \\
(20-55)\end{array}$ & 0.03 \\
\hline $\mathrm{DBP}(\mathrm{mmHg})$ & $\begin{array}{c}59.3^{*} \\
(18.7-42.4)\end{array}$ & $\begin{array}{c}61.9 \\
(20-65)\end{array}$ & 0.30 & $\begin{array}{c}59.3 \pm 11.9^{\dagger} \\
(37.5-90)\end{array}$ & $\begin{array}{c}65.4 \pm 15.4 \\
(40-105)\end{array}$ & 0.05 & $\begin{array}{c}59.72 \pm 10.9^{\dagger} \\
(37.5-80)\end{array}$ & $\begin{array}{c}67.7 \pm 15.0 \\
(45-105)\end{array}$ & 0.02 \\
\hline $\begin{array}{l}\text { Fasting glucose } \\
(\mathrm{mg} / \mathrm{dL})\end{array}$ & $\begin{array}{l}96.5 \pm 15.0^{\dagger} \\
(61.9-126.4)\end{array}$ & $\begin{array}{c}92.2 \pm 14.4 \\
(49.8-119)\end{array}$ & 0.11 & $\begin{array}{c}93.70 \pm 14.0^{\dagger} \\
(49.8-120.7)\end{array}$ & $\begin{array}{c}96.6 \pm 14.1 \\
(79.3-122.8)\end{array}$ & 0.32 & $\begin{array}{c}94.53 \pm 14.3^{\dagger} \\
(52.6-122.8)\end{array}$ & $\begin{array}{l}97.0 \pm 17.0 \\
(24.4-71.9)\end{array}$ & 0.47 \\
\hline Insulin (mU/L) & $\begin{array}{c}7.56 \pm 5.4^{\dagger} \\
(0-24.4)\end{array}$ & $\begin{array}{c}7.74 \pm 6.33 \\
(0-38.9)\end{array}$ & 0.87 & $\begin{array}{c}7.2^{*} \\
(6.3-38.9)\end{array}$ & $\begin{array}{c}6.2 \\
(8-25.4)\end{array}$ & 0.31 & $\begin{array}{c}7.02^{*} \\
(5.3-25.4)\end{array}$ & $\begin{array}{c}7.5 \\
(6.4-17.2)\end{array}$ & 0.37 \\
\hline HOMA & $\begin{array}{c}1.5 \\
(1.8-6.8)\end{array}$ & $\begin{array}{c}1.5 \\
(1.8-10.2)\end{array}$ & 0.59 & $\begin{array}{c}1.61^{*} \\
(1.6-10.2)\end{array}$ & $\begin{array}{c}1.57 \\
(1.8-6.6)\end{array}$ & 0.60 & $\begin{array}{c}1.56 \\
(1.32-6.6)\end{array}$ & $\begin{array}{c}1.79 \\
(2.2-4.4)\end{array}$ & 0.24 \\
\hline $\mathrm{AN}, \mathrm{n}(\%) * *$ & $0(0)$ & $8(8.9)$ & 0.04 & $6(6.4)$ & $2(11.7)$ & 0.48 & $3(4.7)$ & $3(12)$ & 0.22 \\
\hline
\end{tabular}

† Mean \pm standard deviation (minimum - maximum). *Median (interquartile range [p25-p75]). Abbreviations: z-BMl/A z: score of body mass index for age, z-H/A z: score height for age, z-W/A z: score weight for age, SBP: systolic blood pressure, DBP: diastolic blood pressure, AN: acanthosis nigricans, WC: waist circumference, HDL-C: high density lipoprotein cholesterol, SBP: systolic blood pressure, DBP: diastolic blood pressure, TC: total cholesterol, LDL-C: low density lipoprotein, VLDL: very low density lipoprotein, HOMA: Homeostasis Model Assessment. 'Difference between groups by two sample Student's t-test and Mann Whitney U Test for non-normal data $(\mathrm{p}<0.05) .{ }^{*}$ Chi-squared test.

Table 3: Anthropometric characteristics and metabolic profile of the total population and categorized by family history of obesity, diabetes, and cardiovascular disease.

\begin{tabular}{|c|c|c|c|}
\hline Criteria & $\begin{array}{c}\text { IDF (2007) for children over } 10 \\
\text { years old } \\
(\%)\end{array}$ & $\begin{array}{c}\text { IDF (2007), NCEP (1991), NHBPEP (2005) } \\
\text { (this study analysis) } \\
(\%)\end{array}$ & $\begin{array}{l}\text { Three or more risk factors NCEP (1991), NHBPEP } \\
\text { (2005) without WC as a condition. } \\
\text { (this study analysis) }\end{array}$ \\
\hline Total & 4.1 & 6.3 & 10.4 \\
\hline \multicolumn{4}{|l|}{ Area } \\
\hline Rural & 2.5 & 5.0 & 10.1 \\
\hline Urban & 5.4 & 7.4 & 10.7 \\
\hline \multicolumn{4}{|l|}{ Sex } \\
\hline Girls & 5.7 & 7.9 & 11.4 \\
\hline Boys & 2.3 & 4.7 & 9.4 \\
\hline
\end{tabular}

Abbreviations: IDF: International Diabetes Federation, NCEP: National Cholesterol Education Program, NHBPEP: National High Blood Pressure Education Program.

Table 4: Proportion (\%) of children with metabolic syndrome according to the different criteria used in this study.

and the differences between groups was not significant $(\mathrm{p}=0.24)$. The second criteria for MetS (Table 5) considered abdominal obesity plus two other risk factors according to the NCEP [30] and NHBPEP [20] percentiles. MetS was found in 17 children (6.3\%), 5.0\% from the rural and $7.4 \%$ from the urban areas, with no significant differences between groups. The third criteria for MetS diagnosis considered children with three or more risk factors for MetS and the presence of abdominal obesity was not obligatory. Of the total population, $10.4 \%$ had MetS, and the same percentage of children with MetS was present in both areas of study (rural $10.1 \%$ vs. urban $10.7 \%, \mathrm{p}=0.86$ ). The reliability analysis using the kappa coefficient $(0.6487, \mathrm{p}=0.000)$ showed that the agreement was substantial in the total population.

\section{Discussion}

This study demonstrates that MetS is present in children aged 6 to 9 years living in urban and rural areas of northwest Mexico. In this study 
Citation: Ramírez-Murillo C, Guillot-Sánchez E, Elizabeth Artalejo-Ochoa QB, Robles-Sardin AE, Ponce-Martínez JA, et al. (2015) Prevalence of Metabolic Syndrome Diagnosed by Three Different Criteria in School-Aged Children from Rural and Urban Areas of Northwest Mexico. $J$ Metabolic Synd 4: 181. doi:10.4172/2167-0943.1000181

\begin{tabular}{|c|c|c|c|}
\hline Indicator & $\begin{array}{l}\text { Without MS } \\
\quad(n=251)\end{array}$ & $\begin{array}{l}\text { With MS } \\
(\mathrm{n}=17)\end{array}$ & $\mathrm{p}^{1}$ \\
\hline Age $\left(\right.$ years) ${ }^{*}$ & $\begin{array}{c}7.5 \\
(1.3-3.9)\end{array}$ & $\begin{array}{c}7.3 \\
(1.45-2.7)\end{array}$ & 0.45 \\
\hline Weight $(\mathrm{Kg})^{*}$ & $\begin{array}{c}24.14 \\
(8.1-31.85)\end{array}$ & $\begin{array}{c}42.69 \\
(9.87-28.8)\end{array}$ & $<0.01$ \\
\hline Height $(\mathrm{cm})^{\dagger}$ & $\begin{array}{c}123.67 \pm 7.24 \\
(123-124.8)\end{array}$ & $\begin{array}{l}129.58 \pm 5.97 \\
(122.7-129.2)\end{array}$ & $<0.01$ \\
\hline $\mathrm{WC}(\mathrm{cm})^{*}$ & $\begin{array}{c}55 \\
(8.8-45.3)\end{array}$ & $\begin{array}{c}78.5 \\
(8.15-27.5)\end{array}$ & $<0.01$ \\
\hline z-BMI/A* & $\begin{array}{c}-0.06 \\
(1.81-6.52)\end{array}$ & $\begin{array}{c}3.37 \\
(1.73-3.52)\end{array}$ & $<0.01$ \\
\hline Body fat $(\%)^{*}$ & $\begin{array}{c}26.37 \\
(9.92-41.73)\end{array}$ & $\begin{array}{c}44.13 \\
(5.12-9.85)\end{array}$ & $<0.01$ \\
\hline $\begin{array}{c}\text { Triglycerides (mg/ } \\
\mathrm{dL})^{*}\end{array}$ & $\begin{array}{c}85.95 \\
(41.93-225.54)\end{array}$ & $\begin{array}{c}138.88 \\
(65.57-301.42)\end{array}$ & $<0.01$ \\
\hline $\mathrm{TC}(\mathrm{mg} / \mathrm{dL})^{*}$ & $\begin{array}{c}169.38 \\
(46.26-191.08)\end{array}$ & $\begin{array}{c}178.31 \\
(43.30-162.1)\end{array}$ & 0.05 \\
\hline $\mathrm{HDL}-\mathrm{C}(\mathrm{mg} / \mathrm{dL})^{*}$ & $\begin{array}{c}49.75 \\
(11.84-96.68)\end{array}$ & $\begin{array}{c}42.35 \\
(10.98-35.57)\end{array}$ & $<0.01$ \\
\hline LDL-C $(\mathrm{mg} / \mathrm{dL})^{\dagger}$ & $\begin{array}{c}100.76 \pm 30.49 \\
(30.2-200.1)\end{array}$ & $\begin{array}{c}117.88 \pm 38.30 \\
(47.9-224.2)\end{array}$ & 0.07 \\
\hline $\operatorname{VLDL}(\mathrm{mg} / \mathrm{dL})^{*}$ & $\begin{array}{c}17.19 \\
(8.39-45.11)\end{array}$ & $\begin{array}{c}27.78 \\
(13.11-60.28)\end{array}$ & $<0.01$ \\
\hline $\mathrm{SBP}(\mathrm{mmHg})^{*}$ & $\begin{array}{c}90 \\
(20-70)\end{array}$ & $\begin{array}{c}115 \\
(20-40)\end{array}$ & $<0.01$ \\
\hline $\mathrm{DBP}(\mathrm{mmHg})^{*}$ & $\begin{array}{c}60 \\
(15-52.5)\end{array}$ & $\begin{array}{c}80 \\
(11.25-55)\end{array}$ & $<0.01$ \\
\hline Glucose $(\mathrm{mg} / \mathrm{dL})^{*}$ & $\begin{array}{c}92.98 \\
(19.51-85.39)\end{array}$ & $\begin{array}{c}100.78 \\
(14.2-55.9)\end{array}$ & 0.27 \\
\hline Insulin $(\mathrm{mU} / \mathrm{L})^{*}$ & $\begin{array}{c}5.7 \\
(5.72-33.79)\end{array}$ & $\begin{array}{c}13.74 \\
(10.89-57.09)\end{array}$ & $<0.01$ \\
\hline HOMA $^{*}$ & $\begin{array}{c}1.34 \\
(1.38-7.18)\end{array}$ & $\begin{array}{c}3.21 \\
(2.27-14.31)\end{array}$ & $<0.01$ \\
\hline AN, $n(\%)^{* *}$ & $7(2.8)$ & $8(47.1)$ & $<0.01$ \\
\hline
\end{tabular}

${ }^{\dagger}$ Mean \pm standard deviation (minimum - maximum). *Median (interquartile range [p25-p75]).

'Difference between groups by two sample Student's t-test and Mann Whitney U Test for non-normal data $(p<0.05) .{ }^{* *}$ Chi-squared test.

Abbreviations: $z-B M I / A$ z: score of body mass index for age, z-H/A z: score height for age, z-W/A z: score weight for age, SBP: systolic blood pressure, DBP: diastolic blood pressure, AN: acanthosis nigricans, WC: waist circumference, HDL-C: high density lipoprotein cholesterol, SBP: systolic blood pressure, DBP: diastolic blood pressure, TC: total cholesterol, LDL-C: low density lipoprotein, VLDL: very low density lipoprotein, HOMA: Homeostasis Model Assessment.

Table 5: Anthropometric characteristics and metabolic profile according to the presences of metabolic syndrome (MS) with abdominal obesity as an obligatory factor (second criteria used in this study).

population, overweight and obesity represented one of the risk factors for MetS. In both the rural and urban areas, the prevalence of obesity was elevated (31.1\% and $37 \%$, respectively). The percentage of urban children with obesity was higher than that reported by the Nutrition and Health National Survey 2012 [32].

Towards the end of the first year of life, a physiological loss of fat mass takes place, so that in normal conditions a child between 5 and 6 years should be physiologically thinner. When a 6-year old is an obese child, the probability of persistent obesity increases by $50 \%$ [33]. Obesity in parents influenced the development of obesity in their children and this influence seemed higher when the child was aged less than 10 years $[33,34]$. In this study, the results from the family history questionnaire on chronic diseases showed that children from the rural area with a family history of cardiovascular diseases and obesity had higher waist circumference. In contrast, children from the urban area with the same family history had higher systolic and diastolic blood pressure (data not shown). We do not have a clear idea of the differences between urban and rural children, as they have similar family history. A possible explanation is that in the urban areas, there is a greater tendency to consume fast foods that typically have a high sodium content, which probably could be affecting the gene expression associated with blood pressure. Kuschnir and Mendoça mentioned that the risk of developing hypertension is higher when both parents have the disease [35].

Beside heredity, other factors that may contribute to the growing prevalence of obesity and its complications in this population are low physical activity and an inadequate diet. Although data on dietary habits and physical activity were not collected. Results from others studies [36-38] in urban locations of the same region showed that children consumed a hypercaloric diet, with adequate quantities of protein and fiber but excessive amounts of simple carbohydrates and saturated fat and a low quantity of polyunsaturated fat. Those studies also showed that most of the children were sedentary $[36,38]$. The development of obesity secondary to genetic or excessive consumption of food has been proposed as a risk factor for developing metabolic alterations including insulin resistance and diabetes mellitus [39]. Transnational food chains have made energy-dense, low-cost food available and accessible to Mexican children in a community environment where food regulation is still scarce [40].

The analysis of the metabolic profile showed that children from the rural area had more alterations in their lipid profile, with higher triglyceride and VLDL-C values than children from the urban area. In contrast, children from the urban area had more alterations in glucose metabolism, with higher concentrations of glucose and insulin and higher HOMA than children from the rural area. These results suggest that children from the urban area at greater risk for developing heart disease or diabetes, possibly due to risk factors related to lifestyle. Studies on the migration of people from rural to urban areas have reported similar changes in body composition and clinical markers associated with their new lifestyle, which involve higher consumption of energy-dense foods and less physical activity $[41,42]$.

A simple analysis of fasting blood glucose does not determine the diagnosis of diabetes, but it may be the first step in its detection. In this study, we classified fasting blood glucose concentrations according to the American Diabetes Association [31]. Of the total population of the study, $31.3 \%$ (21\% rural, $38.3 \%$ urban) of children had impaired fasting blood glucose. These children are probably producing more insulin to compensate for the elevated glucose, but the two are not balancing each other out [43]. We could assume that this situation has progressed for some time and could turn into T2DM in the future.

T2DM begins with reduced insulin action due to an impairment in the ability of the target cells to respond to this hormone, which leads to insulin resistance. During this process, there is a decrease in the peripheral consumption of glucose and also in hepatic glycogenesis, and an increase in glyconeogenesis with results in hyperglycemia. Also, $\beta$ cells from the pancreatic islet cells hypersecrete insulin to compensate for the excess glucose, producing hyperinsulinemia and normoglycemia. When the cycle is exhausted, it leads to glucose intolerance and, in later stages to T2DM $[43,44]$.

We found children with insulin resistance in both areas $(7.5 \%$ rural, $14.8 \%$ urban, $\mathrm{p}=0.06$ ). Even when we did not detect significant differences between groups, the proportion of children with insulin resistance was higher in the urban area. Secondary to obesity, insulin resistance may be a common etiopathogenic mechanism for hypertension and dyslipidemia. Generally, children with obesity have a higher prevalence of hypertriglyceridemia and low levels of HDL-C [45]. In adipose tissue, hyperinsulinemia increases lipolysis, leading to 
Citation: Ramírez-Murillo C, Guillot-Sánchez E, Elizabeth Artalejo-Ochoa QB, Robles-Sardin AE, Ponce-Martínez JA, et al. (2015) Prevalence of Metabolic Syndrome Diagnosed by Three Different Criteria in School-Aged Children from Rural and Urban Areas of Northwest Mexico. $J$ Metabolic Synd 4: 181. doi:10.4172/2167-0943.1000181

Page 7 of 9

more availability of free fatty acids, an increase in the hepatic synthesis of VLDL, and a decrease in HDL-C [46].

AN was found only in children with high body fat mass, and this coincided with insulin resistance being present only in children with high fat mass. The prevalence of high SBP and/or DBP was $16 \%$ rural vs $11.4 \%$ urban, the presence of hypertension at an early age is might be an early sign of glucose intolerance [47] and a risk factor for coronary disease [48]

Another risk factor for coronary disease is dyslipidemia. In this study, $32.1 \%$ of children had hypertriglyceridemia and $19 \%$ had low HDL-C. Other studies in Sonoran children reported low levels of HDL-C and the predominant subfraction was $\mathrm{HDL}_{3}$, which is associated with higher cardiovascular risk [38]. Obesity, hypertension, hypertriglyceridemia, low HDL-C, and impaired glucose are components of MetS.

One of the most important goals of this study was to evaluate the prevalence of MetS in children using three different diagnostic criteria and determine which of them might be more appropriate for the diagnosis of MetS in a pediatric population. The first criteria used for the diagnosis of MetS in this study were the one according to the IDF [4] for 10-year-old children. These criteria use cutoffs for adults for the biochemical indicators and account for the presence of waist circumference $\geq 90^{\text {th }}$ percentile for the diagnosis of MetS. The general prevalence of MetS using these criteria was $4.1 \%(2.5 \%$ rural; 5.4\% urban). The IDF does not consider the presence of MetS in children aged $<10$ years and does not recommend its diagnosis unless the children have a family history of risk factors [4]. This study demonstrates that even when criteria for adults is used, there are children with MetS in the region of Sonora. A study by ElizondoMontemayor et al. [49] using the IDF criteria [4] in overweight children age 6 to 12 years from Mexico City reported a prevalence of MetS of $6.7 \%$. When they observed age groups of 6-9 years and $10-12$ years, the prevalence of MetS was 7.3\% and 5.9\% ( $\mathrm{p}=0.91$ ), respectively. These authors mentioned that there were more children with MetS in the younger age group, and this may be due to the fact that the percentage of children with waist circumference above the $90^{\text {th }}$ percentile [4] was significantly higher $(p<0.001)$ in the group of children aged 6-9 years compared with the older age group.

Another study done in Campeche, México, used the same IDF criteria [4] and reported a prevalence of $20 \%$ MetS in adolescents aged 11 to 13 years. Nevertheless, only obese participants (BMI $\geq 95^{\text {th }}$ percentile) were included [3].

The second way in which we determined the prevalence of MetS also considered the IDF criteria [4] using a waist circumference $\geq 90^{\text {th }}$ percentile as a condition for the diagnosis, but also employed adequate cutoffs for metabolic measurements in children aged 6 to 9 years provided by the NCEP [30] for the lipid profile, and by the NHBPEP [20] for blood pressure. Thus, the observed prevalence of MetS in the total study population was $6.3 \%$ (5.0\% rural vs. $7.4 \%$ urban, $\mathrm{p}=0.43)$. Thus, the ratio obtained was similar to that reported by ElizondoMontemayor et al. (7.3\%) in obese children aged 6-9 years [49]. However, they used the IDF definition [4] without adjusting the cutoffs for children. Differences in physical and metabolic characteristics between children with and without the presence of MetS were evident (Table 5). Children with MetS had higher values in all the body composition variables and metabolic risk factors, and approximately $50 \%$ of children diagnosed with MetS presented AN. The consideration of central obesity plus the appropriate clinical cutoffs for children, could be a better criterion for evaluating MetS in a pediatric population
The third way to diagnose MetS did not take into account the presence of a waist circumference $\geq 90^{\text {th }}$ percentile as a condition for evaluating MetS and used lipid profile metabolic indicator cutoffs for children aged 6 to 9 years from the NCEP and blood pressure criteria from the NHBPEP. Thus, the prevalence of MetS using these criteria was $10.4 \%$ (10.1\% rural vs. $10.7 \%$ urban), and $32.1 \%$ of the children presented with $\mathrm{AN}$. When we diagnosed MetS this way, children with a normal body composition met this criterion because other risk factors like impaired fasting glucose, hypertension, hypertriglyceridemia, or low HDL-C were present. Even without considering obesity, some children may have altered metabolic factors, possibly due to genetic factors that put children at risk. High levels of TC, LDL-C, plasma glucose, and high blood pressure are risk factors that could be influenced by family history due to shared genetic and environmental factors [5].

Considering central obesity as the major factor for the diagnosis of MetS may underestimate the risk of developing cardiovascular diseases and T2DM because it excludes children without obesity who have other metabolic alterations.

The proportions of MetS identified in this population of children varied depending on the criteria used. We hypothesize that the IDF [4] criteria could underestimate the prevalence of MetS in children aged less than 10 years because the cutoffs for the lipid profile and blood pressure are not adequate for this age group. Instead, the second criteria included cutoffs suggested for this age from the NCEP [30] and the NHBPEP [20], which may detect more accurately children with MetS. The third criteria for evaluation of MetS included children with a normal waist circumference but who presented with three other risk factors. This third way of diagnosing MetS could be useful for identifying children at risk for cardiovascular and T2DM due to heredity factors.

We recognize that one of the limitations of this study was that we did not collect data on dietary habits and physical activity as factors related to obesity; however, data from recent studies were available for a proper discussion. The main strength of this study is that we measured MetS in children aged less than 10 years from rural and urban areas. We established three different criteria for evaluating MetS in children, and all the techniques used were applied by trained personnel.

\section{Conclusions}

This study demonstrates that MetS is present in children aged 6 to 9 years living in a community with serious problems of obesity and cardiovascular diseases. We also demonstrate that MetS is more prevalent in overweight and obese children. The second criteria used in this study could be most suitable for diagnosis of MetS in a pediatric population because it takes into account the appropriate cutoffs suggested for this age. Considering abdominal obesity as an obligatory factor for the diagnosis of MetS could underestimate the number of children at risk for cardiovascular diseases and T2DM because the criteria exclude children without abdominal obesity but who do have metabolic alterations. Therefore, MetS should be diagnosed using an age-appropriate definition. Our information suggests that metabolic problems in children from an urban area are at increased risk for heart disease and diabetes compared to the rural area.

Because obesity is associated risk factors for cardiovascular diseases and T2DM, health promotion programs should consider the combination of genetic and environmental factors. If these factors are detected and controlled in time, the development of non-communicable diseases related to MetS could be prevented. 
Citation: Ramírez-Murillo C, Guillot-Sánchez E, Elizabeth Artalejo-Ochoa QB, Robles-Sardin AE, Ponce-Martínez JA, et al. (2015) Prevalence of Metabolic Syndrome Diagnosed by Three Different Criteria in School-Aged Children from Rural and Urban Areas of Northwest Mexico. $J$ Metabolic Synd 4: 181. doi:10.4172/2167-0943.1000181

\section{Implications for School Health}

The development of metabolic syndrome or related-conditions including obesity, hypertension, cardiovascular diseases, and type 2 diabetes mellitus in school-aged children could interfere with their adequate physical and mental development. The diagnostic criteria evaluated in this study may be useful to develop a useful definition for clinical and epidemiological practices of pediatric population in risk of chronic diseases.

\section{Human Subjects Approval Statement}

This research was approved by the Ethics Committee of the Food and Development Research Center (CIAD) (Document code $\mathrm{CE} / 018 / 2009)$

\section{Acknowledgments}

We thank the children and their parents for their participation. We also thank the School Breakfast Program of Sonora (DIF, Sonora) for supporting this study.

\section{References}

1. Alberti G, Zimmet P, Shaw J (2006) Metabolic syndrome- a new world-wide definition. A Consensus Statement from the International Diabetes Federation. Diabet Med 23: 469-480.

2. Gami A, Witt BJ, Howard D, Erwin P, Gami L, et al. (2007) Metabolic syndrome and risk of incident cardiovascular events and death: a systematic review and meta-analysis of longitudinal studies. J Am Coll Cardiol 49: 403-414.

3. Juárez-López $C$, Klunder-Klunder $M$, Medina-Bravo $P$, Madrigal-Azcarate A, Mass-Diaz E, et al. (2010) Insulin resistance and its association with the components of the metabolic syndrome among obese children and adolescents. BMC Public Health 10: 318

4. Zimmet P, Alberti G, Kaufman F, Tajima N, Silink M, et al. (2007) The metabolic syndrome in children and adolescents: the IDF consensus. Diabetes Voice 52: 29-32.

5. Steinberger J, Daniels SR, Eckel RH, Hayman L, Lustig RH, et al. (2009) Progress and challenges in metabolic syndrome in children and adolescents: A scientific statement from the American Heart Association Atherosclerosis, Hypertension, and Obesity in the Young Committee of the Council on Cardiovascular Disease in the Young; Council on Cardiovascular Nursing; and Council on Nutrition, Physical Activity, and Metabolism Circulation 119: 628647

6. Nguyen QM, Srinivasan SR, Xu JH, Chen W, Berenson GS (2008) Changes in risk variables of metabolic syndrome since childhood in pre-diabetic and type 2 diabetic subjects. Diabetes Care 31: 2044-2049.

7. Daniels SR, Greer F (2008) Committee on Nutrition Lipid screening and cardiovascular health in childhood. Pediatrics 122: 198-208.

8. Ayaz T, Baydur Azahin S, Sahin OZ (2014) Relation of acanthosis nigricans to metabolic syndrome in overweight and obese women. Metab Syndr Rela Disord 12: 320-323.

9. Garg MK, Dutta MK, Brar KS (2012) Inflammatory markers in metabolic syndrome. Int J Diabetes Dev Ctries 32: 131-137.

10. Secretaría de Salud (2007) Programa Nacional de Salud 2007-2012 Por un México sano: construyendo alianzas para una mejor salud. Pp. 28-29.

11. Morrison J, Glueck C, Horn P, Wang P (2010) Childhood predictors of adult type 2 diabetes at 9- and 26-year follow-ups. Arch Pediatr Adolesc Med 164 53-60.

12. Morrison JA, Friedman LA, Gray-McGuire C (2007) Metabolic syndrome in childhood predicts adult cardiovascular disease 25 years later: The Princeton lipid research clinics follow-up study. Pediatrics 120: 340-345.

13. INSP (2013) Encuesta Nacional de Salud y Nutrición 2012. Resultados por entidad federativa, Sonora. Cuernavaca, México, Instituto Nacional de Salud Pública.

14. Steinberger J, Daniels SR (2003) Obesity, Insulin Resistance, Diabetes, and Cardiovascular Risk in Children: An American Heart Association Scientific Statement From the Atherosclerosis, Hypertension, and Obesity in the Young Committee (Council on Cardiovascular Disease in the Young) and the Diabetes
Committee (Council on Nutrition, Physical Activity, and Metabolism). Circulation 107:1448-1453.

15. Barlow SE, Dietz WH (1998) Obesity evaluation and treatment: Expert Committee Recommendations. Pediatrics 102: E29.

16. Villalvazo P, Corona JP, García S (2002) Urbano-rural, constante búsqueda de fronteras conceptuales. Notas, revista de información y análisis 20: 17-24.

17. WHO (2010) Anthro for personal computers version 3.2.2, 2011: Software for assessing growth and development of the world's children Geneva, World Health Organization.

18. Ramírez-Lopez E, Grijalva-Haro MI, Valencia ME, Ponce JA Artalejo E (2005) Impacto de un programa de desayunos escolares en la prevalencia de obesidad y factores de riesgo cardiovascular en niños sonorenses. Salud Publica Mex 47: 126-133.

19. Freedman D, Wang J, Thornton J, Mei Z, Sopher A, et al. (2009) Classification of body fatness by body mass index-for-age categories among children. Arch Pediatr Adolesc Med 163: 805-811.

20. NHBPEP (2005) National High Blood Pressure Education Program Working Group on High Blood Pressure in Children and Adolescents. The fourth report on the diagnosis, evaluation, and treatment of high blood pressure in children and adolescents. Bethesda, MD, National Insitutes of Health.

21. Higgins SP, Freemark M, Prose NS (2008) Acanthosis nigricans: a practica approach to evaluation and management. Dermatol Online J 14 :2.

22. Allain CC, Poon LS, Chan CSG, Richmond W, Fu PC (1974) Enzymatic determination of total serum cholesterol. Clin Chem 20: 470-475.

23. Warnick GR, Benderson J, Albers JJ (1982) Dextran sulfate-Mg2+ precipitation procedure for quantitation of high-density-lipoprotein cholesterol. Clin Chem 28(6): 1379-1388

24. Friedewald WT, Levy RI, Fredrickson DS (1972) Estimation of the concentration of low-density lipoprotein cholesterol in plasma, without use of the preparative ultracentrifuge. Clin Chem 18: 499-502.

25. Siedel J, Schmuck R, Staepels J (1993) Long-term stable liquid ready-to-use monoreagent for the enzimatic assay of serum or plasma triglicerides (GPO PAP method), AACC Meeting (Abstract 34). Clin Chem 39: 1127.

26. Trinder $P$ (1969) Determination of blood glucose using an oxidase-peroxidase system with a non-carcinogenic chromogen. J Clin Pathol 22 :158-161.

27. Matthews DR, Hosker JP, Rudenski AS, Naylor BA, Treacher DF, et al. (1985) Homeostasis model assessment: insulin resistance and beta-cell function from fasting plasma glucose and insulin concentrations in man. Diabetologia 28 412-419.

28. Garcia-Cuartero B Garcia-Lacalle C Jimenez-Lobo C, Gonzalez-Vergaz A Calvo-Rey C, et al. (2007) Indice HOMA y QUICKI, insulina y péptido C en niños sanos. Puntos de corte de riesgo cardiovascular. An Pediatr (Barc.) 66 481-490.

29. Williams CL, Hayman LL, Daniels SR, Robinson TN, Steinberger J, et al. (2002) Cardiovascular health in childhood: A statement for health professionals from the Committee on Atherosclerosis, Hypertension, and Obesity in the Young (AHOY) of the Council on Cardiovascular Disease in the Young. American Heart Association. Circulation 106: 143-160.

30. NCEP (1991) National Cholesterol Education Panel. The Expert Panel On Blood Cholesterol Levels In Children and Adolescents. Nutrition Today 26: 36 41.

31. ADA (2012) American Diabetes Association Standards of medical care in diabetes-2012. Diabetes Care 35: S11-S63.

32. INSP (2007) Encuesta Nacional de Salud y Nutrición 2006. Resultados po entidad federativa, Sonora. Cuernavaca, México, Instituto Nacional de Salud Pública-Secretaria de Salud.

33. Moran R (1999) Evaluation and treatment of childhood obesity. Am Fam Physician 59(4):861-868

34. Whitaker R, Wright J, Pepe M, Seidel K, Dietz W (1997) Predicting obesity in young adulthood from childhood and parental obesity. N Engl J Med 337 869-873.

35. Kuschnir MC, Mendoça GA (2007) Risk factors associated with arteria hypertension in adolescents. J Pediatr 83: 335-342.

36. Ballesteros MN, Cabrera RM, del Socorro Saucedo M, Fernandez ML (2004) 
Citation: Ramírez-Murillo C, Guillot-Sánchez E, Elizabeth Artalejo-Ochoa QB, Robles-Sardin AE, Ponce-Martínez JA, et al. (2015) Prevalence of Metabolic Syndrome Diagnosed by Three Different Criteria in School-Aged Children from Rural and Urban Areas of Northwest Mexico. $\mathrm{J}$ Metabolic Synd 4: 181. doi:10.4172/2167-0943.1000181

Dietary cholesterol does not increase biomarkers for chronic disease in a pediatric population from northern Mexico. Am J Clin Nutr 80: 855-861.

37. Enríquez-Leal MC, Montano-Figueroa CA, Saucedo-Tamayo MS, Vidal Ochoa M, Rivera-Icedo BM, et al. (2010) Incidencia, características clínicas y estado nutricional en niños y adolescentes mexicanos con diabetes. Interciencia 35: $455-460$

38. Ballesteros-Vásquez MN, Amaya M, Guerrero EV, García Vedugo K, GrijalvaHaro MI, et al. (2012) Patrón de predominancia de las subfracciones de la lipoproteína HDL y su asociación con riesgo cardiovascular en niños escolares. In Congreso de la Sociedad Latinoamericana de Nutrición SLAN, Ed. Habana, Cuba, Sociedad Latinoamericana de Nutrición, pp. 251.

39. van Vliet M, Heymans M, von Rosenstiel I, Brandjes D, Beijnen J, et al. (2011) Cardiometabolic risk variables in overweight and obese children: a worldwide comparison. Cardiovasc Diabetol 10: 106.

40. Hawkes C (2006) Uneven dietary development: linking the policies and processes of globalization with the nutrition transition, obesity and diet-related chronic diseases. Global Health 2: 4.

41. Hujava Z, Lesniakova M (2011) Anthropometric risk factors of aterosclerosis: Differences between urban and rural East-Slovakian children and adolescents. Bratisl Lek Listy 112: 491-496.

42. Hernandez-Valero MA, Bustamante-Montes LP, Hernández M, Halley-Castillo E, Wilkinson AV, et al. (2012) Higher risk for obesity among Mexican-American and Mexican immigrant children and adolescents than among peers in Mexico. J Immigr Minor Health 14: 517-522.

43. Wilcox G (2005) Insulin and Insulin Resistance. Clinical Biochemist Reviews 26: 19-39.

44. Barja S, Arteaga A, Acosta A (2003) Resistencia insulínica y otras expresiones del síndrome metabólico en niños obesos chilenos. Rev Med Chil 131: 259268.

45. Chiarelli F, Marcovecchio ML (2008) Insulin resistance and obesity in childhood Eur J Endocrinol 159: S67-S74.

46. Cruz ML, Weigensberg MJ, Huang TTK, Ball G, Shaibi GQ, et al. (2004) The metabolic syndrome in overweight hispanic youth and the role of insulin sensitivity J Clin Endocrinol Metab 89: 108-113.

47. Yanes-Quesada M, Perich-Amador P, González-Suárez R, Yanes-Quesada M, Cruz-Hernández J, et al. (2007) Factores clínicos relacionados con la hipertensión arterial en pacientes con trastornos de tolerancia a los carbohidratos. Rev Cubana Med Gen Integ 23

48. Luma GB, Spiotta RT (2006) Hypertension in children and adolescents. Am Fam Physician 73: 1558-1568.

49. Elizondo-Montemayor L, Serrano-Gonzalez M, Ugalde-Casas PA, CuelloGarcia C, Borbolla-Escoboza JR (2010) Metabolic syndrome risk factors among a sample of overweight and obese Mexican children. J Clin Hypertens 12: $380-387$ 